\title{
Modelling the viscoelastic properties of coagulating blood
}

\author{
P. R. Williams ${ }^{1}$, R. L. Williams ${ }^{1}$, K. Hawkins ${ }^{1}$, C. Wright ${ }^{1}$, \\ A. Evans ${ }^{2} \&$ H. Simpkin ${ }^{3}$ \\ ${ }^{I}$ School of Engineering, University of Wales Swansea, Swansea, U.K. \\ ${ }^{2}$ Medical School, University of Wales Swansea, Swansea, U.K \\ ${ }^{3}$ NHS Trust Morriston, Swansea, U.K.
}

\begin{abstract}
The rheological behaviour of coagulating human blood has been measured using a multiple frequency rheometrical technique known as Fourier Transform Mechanical Spectroscopy (FTMS). The results indicate that coagulating blood, prior to the point of incipient clot formation, can be modelled by a modified form of the Gross-Marvin 'ladder' model, and the benefits of such modelling for blood coagulation are discussed.
\end{abstract}

Keywords: rheology, blood coagulation, discrete relaxation spectra.

\section{Introduction}

A comprehensive understanding of the relationships between the microstructure of blood clots and their viscoelastic properties has been a goal of clinical and scientific research for more than 50 years. This goal remains largely unrealised, due to the difficulty of describing the complex gel microstructure of blood clots in conventional morphological terms, and a lack of appropriate rheological techniques. The significance of this issue arises from findings that various pathologies are associated with changes in the viscoelasticity of blood clots [1]. The present inability to relate these changes to underlying microstructural changes prevents the development of a sufficient understanding of the processes involved.

The most widely used rheological instrument used to study blood coagulation is an oscillating-cylinder rheometer called a thromboelastograph (TEG). Since 1948 , it has been used to monitor clot 'rigidity' in terms of cylinder displacement 
(measured in millimeters) from a chart record [2]. TEG measurements are useful in some clinical settings but transgress the non-linear viscoelastic regime during coagulation, thereby substantially modifying clot rheology [3]. More sophisticated rheometry has been employed in haemorheological work, but a feature of most studies is the use of an arbitrary strain amplitude and a single frequency of oscillation. The results of such studies have been variously described in terms of rheological models such as the Maxwell fluid, KelvinVoigt viscoelastic solid or elastic solid [2] but these may be substantially improved upon in terms of representing the properties of coagulating blood.

In this paper we report experimental work in which the coagulation of samples of whole human blood has been monitored up to the point of incipient clot formation using a rheometrical technique known as Fourier Transform Mechanical Spectroscopy (FTMS) [4]. FTMS tests employ several test frequencies simultaneously, the technique being particularly useful in the characterisation of viscoelastic systems (such as coagulating blood) whose rheology changes progressively, as a function of time. The results have been modelled using a modified form of the 'ladder' model proposed by Gross and Marvin [5], and the benefits of its application to coagulating blood are described herein.

\section{Theory and modelling}

The results of small amplitude oscillatory shear tests conducted on linear viscoelastic systems may be represented in terms of the frequency dependent complex shear modulus $G^{*}(\omega)$ and its components, the dynamic rigidity $\left(G^{\prime}(\omega)\right)$ and the loss modulus $\left(\mathrm{G}^{\prime \prime}(\omega)\right)$, respectively. In terms of the generalised Maxwell model, $\mathrm{G}^{*}(\omega)$ is represented as [5];

$$
\mathrm{G}^{*}(\omega)=\left\{\mathrm{G}_{\mathrm{e}}\right\}+\Sigma \mathrm{G}_{\mathrm{n}} . \mathrm{i} \omega \tau_{\mathrm{n}} /\left[1+\mathrm{i} \omega \tau_{\mathrm{n}}\right]
$$

The generalised Maxwell model incorporates a discrete relaxation time spectrum involving relaxation times, $\tau_{n}$, and corresponding moduli, $G_{n}$. In the case of viscoelastic liquids, the equilibrium modulus, $\mathrm{G}_{\mathrm{e}}$, is zero.

Although some workers have attempted to represent blood using the Maxwell model, the results obtained in the present study suggest that a better representation of the evolution of viscoelastic behaviour in coagulating blood may be obtained using 'ladder models' [5]. In discrete forms of these models, viscoelastic elements are associated with discrete nodes, a concept appropriate to a pre- gel point situation which, physically, involves discrete molecular gel clusters. Thus finite versions of these discrete nodal networks may be used to simulate aspects of pre-gel rheological behaviour (the blood clot capable of performing the haemostatic function being formed at the gel point which is represented by their extension to infinite networks [5]). Equivalently, the GrossMarvin series-parallel models may be employed to generate distributions of relaxation times and moduli $\left(\tau_{n}\right.$ and $\left.G_{n}\right)$ in the generalised Maxwell model. 
The following modified Gross-Marvin Model (MGMM hereafter) provides different distributions of the shear moduli (or relaxation 'strengths') $G_{n}$, depending on the value of $\alpha$, which share a common distribution of relaxation times, $\tau_{\mathrm{n}}$, where :

$$
\mathrm{G}_{\mathrm{n}} \sim 1 /(2 \mathrm{~N}+1)\left[\cos (\pi(2 \mathrm{n}+1) /(2(2 \mathrm{~N}+1))]^{2} \cdot \mathrm{n}^{(1-\beta)}\right.
$$

with $\beta=(1-2 \alpha)$ and

$$
\tau_{\mathrm{n}} \sim[\operatorname{cosec}(\pi(2 \mathrm{n}+1) /(2(2 \mathrm{~N}+1))]
$$

yielding the dynamic moduli of the generalised Maxwell model as;

and

$$
\underset{\mathrm{n}=1}{\mathrm{~N}-1} \sim \sum \mathrm{G}_{\mathrm{n}} \omega^{2} \tau_{\mathrm{n}}{ }^{2} /\left(1+\omega^{2} \tau_{\mathrm{n}}{ }^{2}\right)
$$

$$
\underset{\mathrm{n}=1 .}{\mathrm{N}-1}, \sim \mathrm{G}_{\mathrm{n}} \omega \tau_{\mathrm{n}} /\left(1+\omega^{2} \tau_{\mathrm{n}}{ }^{2}\right)
$$

\section{Experimental: materials and methods}

Two test systems were studied using a Rheometrics ARES rheometer fitted with a $50 \mathrm{~mm}$ diameter, 0.04 radian cone-plate geometry (the lower plate temperature being controlled by means of an external waterbath) and a single gap coaxial cylinder geometry with a $1 \mathrm{~mm}$ shearing gap. The first test system chosen for study was an aqueous gelatin solution formed by the dispersal of gelatin powder (provided by Sanofi Bio Industry, France) at $25^{\circ} \mathrm{C}$ to a concentration of $10 \% \mathrm{w} / \mathrm{w}$ in deionised water obtained from a two-stage reverse osmosis, ion-exchange purification system (Elgastat Spectrum, Elga UK). The system was treated with sodium azide as an antimicrobial agent. Samples were heated to $60^{\circ} \mathrm{C}$ and held for 45 minutes prior to rheological tests. The second system consisted of fresh samples of whole human blood taken from healthy volunteers.

For work involving the cone and plate system the gelatin solution, which was initially heated to a temperature of $60^{\circ} \mathrm{C}$, was loaded onto the rheometer's lower platen and harmonically varying displacement waveforms (corresponding to a maximum strain amplitude of $20 \%$ ) were subsequently applied over a frequency range of $0.1 \mathrm{~Hz}$ to $10 \mathrm{~Hz}$ at various temperatures, T. An example of the results obtained at $\mathrm{T}=28^{\circ} \mathrm{C}$ are shown in fig. 1 . Throughout the experiments, including those conducted at $\mathrm{T}=37^{\circ} \mathrm{C}$ on samples of coagulating blood (see fig. 2), the recorded torque waveforms were inspected using Fourier analysis for extraneous harmonic frequencies, in order to verify that measurements were being conducted within the linear viscoelastic regime. Repeat experiments were also conducted using different measuring geometries, the latter verifying the absence of wall-slip phenomena. 


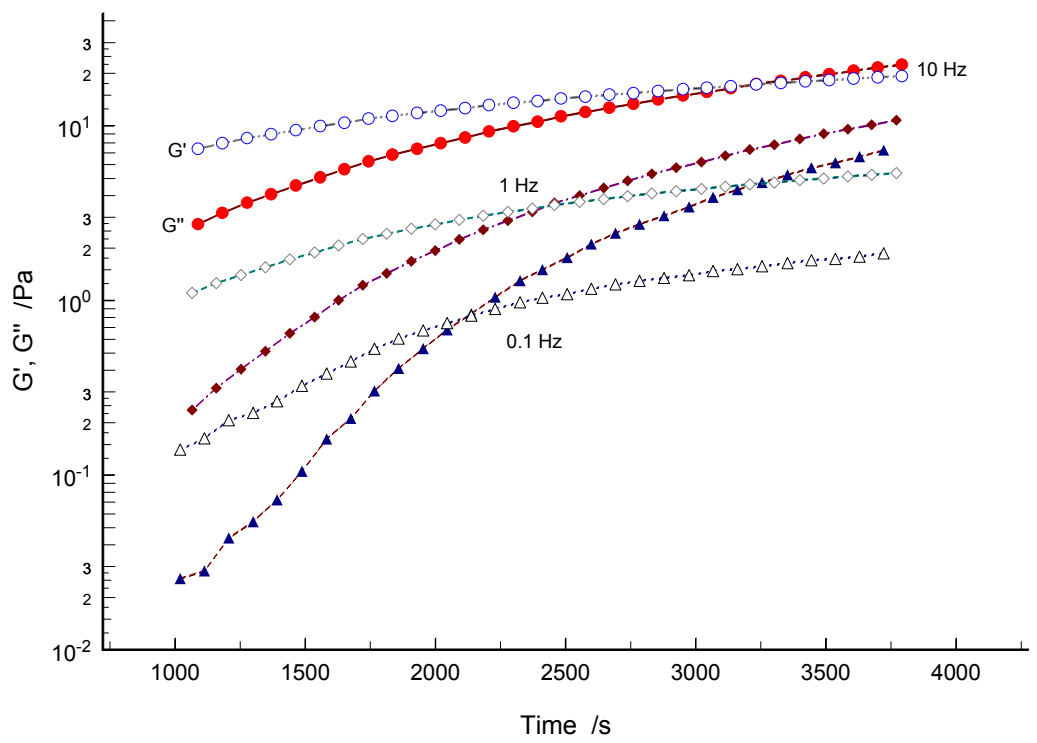

Figure 1: Evolution of storage modulus, G' (open symbols), and loss modulus, G" (closed symbols) at various frequencies for $10 \%$ gelatin solution at $28^{\circ} \mathrm{C}$.

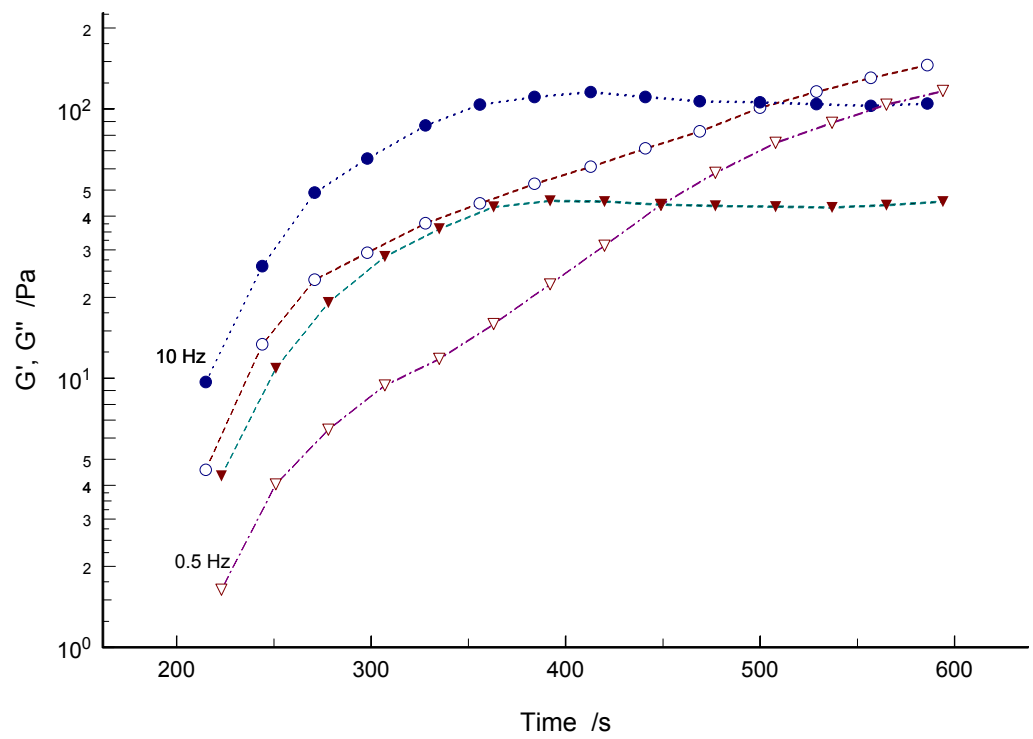

Figure 2: Evolution of storage modulus, G' (open symbols), and loss modulus, G” (closed symbols) at $0.5 \mathrm{~Hz}$ (inverted triangles) and $10 \mathrm{~Hz}$ (circles) for coagulating human blood at $37^{\circ} \mathrm{C}$. 


\section{Results and discussion}

The results obtained from the rheometrical tests on gelatin and blood are shown in figs. 1 and 2, respectively, in terms of the temporal evolution of G' $(\omega)$ and the loss modulus G' $(\omega)$. The evolution of the discrete MGMM network is shown in fig. 3, in terms of the frequency dependence of the loss tangent $\left(\tan \delta=G{ }^{\prime} / G^{\prime}\right)$ for $\alpha=0.20,0.50,0.67$ and 0.90 . As the discrete relaxation time spectra of the networks share a common distribution of relaxation times, irrespective of the value of $\alpha$, the differences in the simulated 'coagulation' behaviour for different $\alpha$ values arise from the different distributions of the corresponding shear moduli, $G_{n}$. Figs. $4(a)$ and $4(b)$ show the relaxation time spectra of networks with $\alpha=0.20,0.50,0.67$ and 0.9 , for $\mathrm{N}=10$ and $\mathrm{N}=20$, respectively. For $\alpha \leq 0.50$ the relaxation spectra are dominated by the lowest frequency modes, with the largest relaxation strength associated with the longest relaxation time. For $\alpha>0.50$, the dominant modes lie at intermediate frequencies, moving to higher frequencies (corresponding to shorter relaxation times) as $\alpha$ increases. This latter feature may be expected, as the systems become increasingly fluid-like in their responses to imposed stresses.
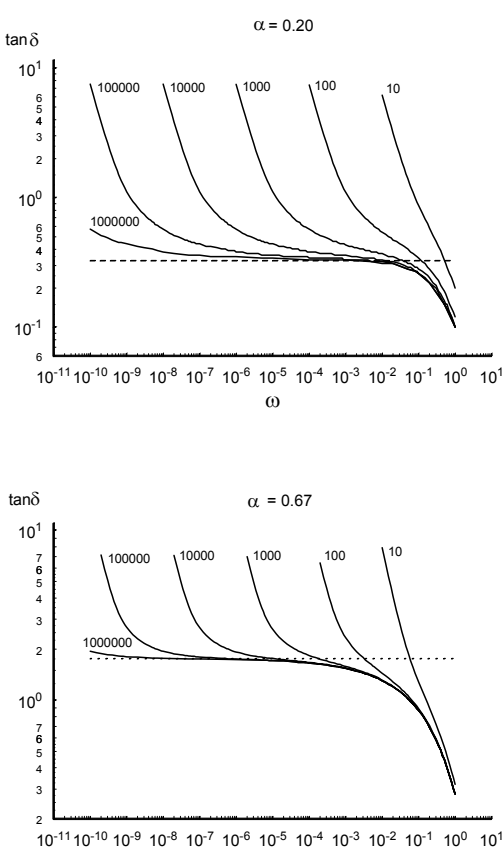

$\omega$

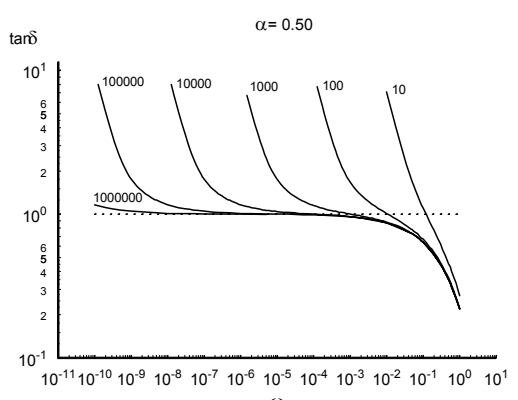

$\omega$

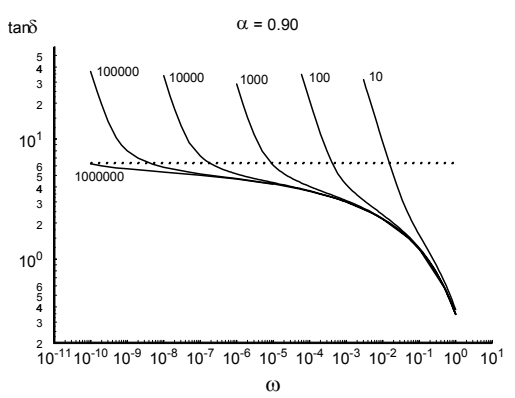

Figure 3: Evolution of viscoelastic properties, in terms of $\tan \delta(\omega)$, for a modified Gross-Marvin model with $\alpha=0.20,0.50,0.67$ and 0.90 . The broken line indicates the frequency independent loss tangent predicted by the Gel Equation for each value of $\alpha$. 
(a)
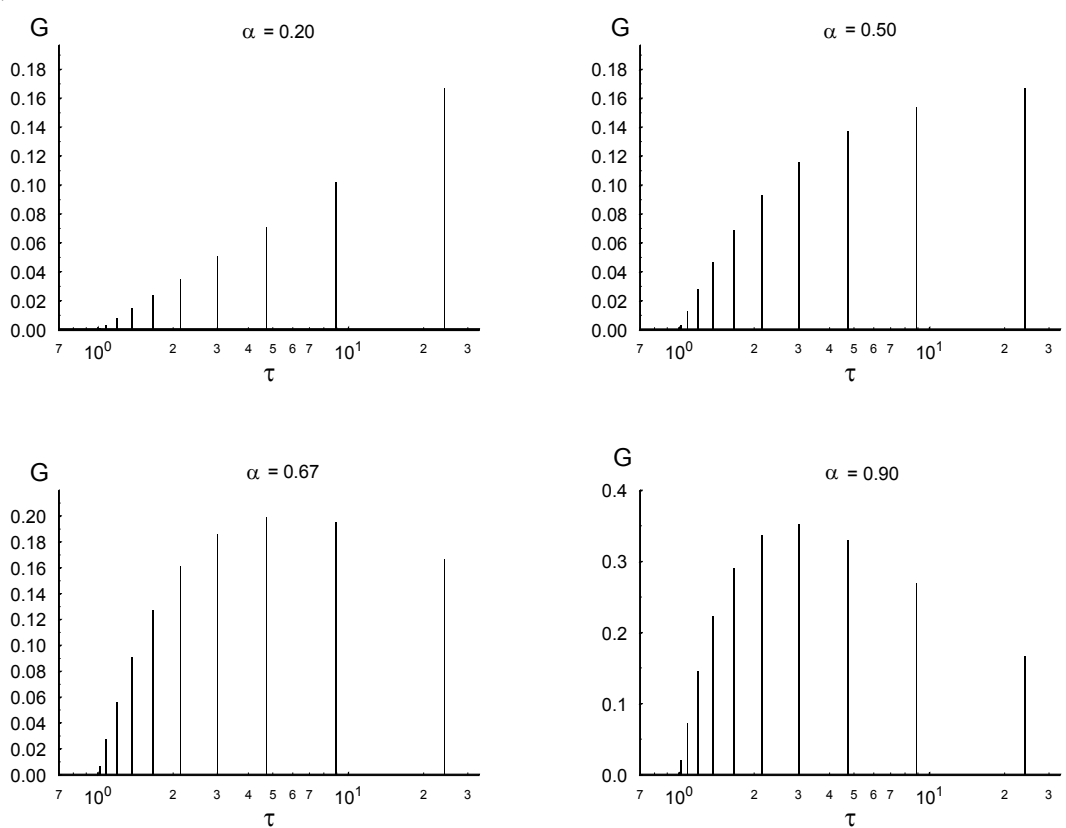

(b)
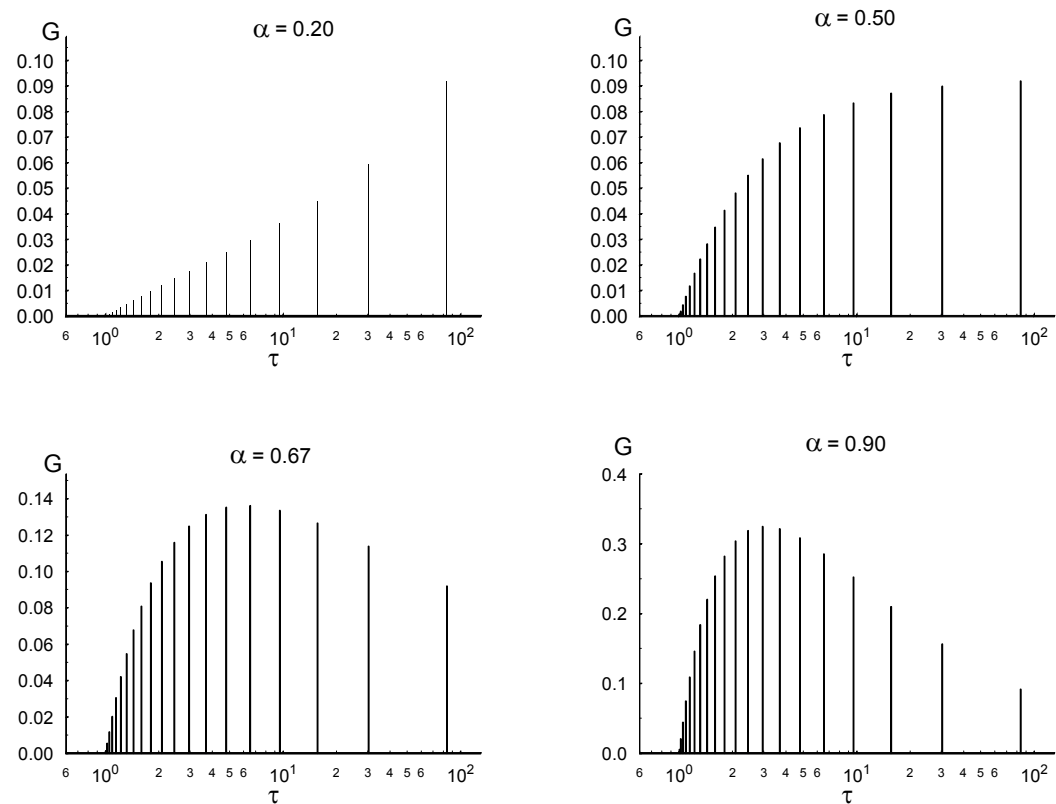

Figure 4: Discrete relaxation spectra for $\alpha=0.20,0.50,0.67$ and 0.90 : (a) 10 nodes ; (b) 20 nodes. 


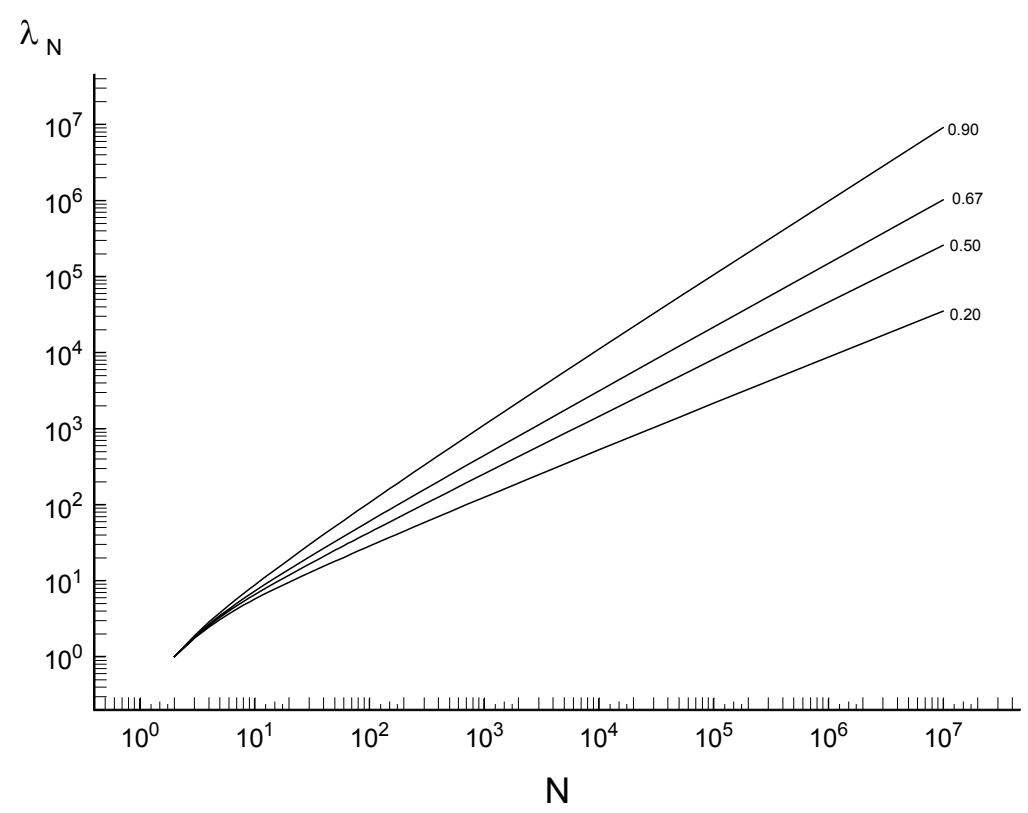

Figure 5: The characteristic wavelength, $\lambda \mathrm{N}$, plotted as a function of the number of nodes, $\mathrm{N}$.

In order to illustrate the growth of the networks we calculate the Fourier Transform of the relaxation time spectra generated by eqns. 2(a,b) which yields the frequency, $\omega_{\mathrm{N}}$. As the number of nodes increases, $\omega_{\mathrm{N}}$ decreases, thereby reflecting an increase in the largest relaxation time. From $\omega_{\mathrm{N}}$ the corresponding wavelength $\lambda_{N}$ is derived, $\lambda_{N}$ being made dimensionless herein by the value of $\lambda$ corresponding to a network of two nodes.

In fig. 5, the wavelength, $\lambda_{\mathrm{N}}$, is plotted as a function of the number of nodes, $\mathrm{N}$. For the sake of clarity, the results shown here involve networks of $10^{7}$ nodes, or less. For $\mathrm{N} \geq 10^{2}$, a single power-law characterises the growth of the networks, the onset of this power-law implying the establishment of a scaleinvariant feature of the corresponding mechanical structure. The results indicate that the macroscopic gel (corresponding to the establishment of the haemostatic blood clot) shares a common structural feature of the smaller networks.

\section{Conclusions}

The results of a rheometrical study of samples of whole human blood using Fourier Transform Mechanical Spectroscopy indicate that the coagulation process may be represented using a modified form of the viscoelastic nodal network known as the modified ladder model. The changing rheological properties of the blood are represented by discrete viscoelastic nodal network elements, a concept appropriate to a pre- gel point situation which, physically, 
involves discrete molecular gel clusters (such as in fibrin gels). Accordingly, finite versions of these discrete nodal networks may be used to simulate aspects of pre-gel rheological behaviour i.e. prior to the establishment at the gel point of the blood clot which is capable of performing the haemostatic function. The latter is represented by the extension of the discrete networks described herein to infinite networks [5].

\section{References}

[1] Thurston GB., Viscoelastic properties of blood and blood analogs, Advances in Hemodynamics and Hemorheology, ed. by T. C. Howe, JAI Press, pp. 1-30, 1996.

[2] Scott Blair GW., An Introduction to Biorheology, Elsevier Scientific, Amsterdam, 1974.

[3] Burghardt WR, Goldstick TK, Leneschmidt J, Kempka K., Nonlinear viscoelasticity and the thrombelastograph .1. Studies on bovine plasma clots, Biorheology, 32, pp. 621-630, 1995.

[4] Holly, E.E., Venkataraman, S.K., Chambon, F. And Winter, H.H., Fouriertransform mechanical spectroscopy of viscoelastic materials with transient structure, J. Non-Newtonian Fluid Mechanics, 27, pp. 17-26, 1988.

[5] NW Tschoegl, The Phenomenological Theory of Linear Viscoelastic Behaviour, Springer, Berlin, 1989. 